\title{
THE HARD ELLIPSOID POTENTIAL MODEL FOR THE ROTATIONAL ENERGY TRANSFER AND ITS REGION IN MOLECULAR SYSTEMS
}

\author{
K. Agrawal ${ }^{a}$, S. TillwankaR ${ }^{b}$ and P. Dashora ${ }^{c}$ \\ ${ }^{a}$ Department of Physics, Government Polytechnic, Ajmer (Raj.), 305 002, India \\ ${ }^{b}$ Department of Physics, Government Polytechnic, Dhar (M.P.), 454 001, India \\ ${ }^{c}$ Department of Physics, University of Rajasthan, Jaipur (Raj.), 302 001, India
}

\section{(Received November 9, 1999; in final form March 14, 2000)}

The hard ellipsoid potential model for understanding the mechanism of the rotational energy transfer in a diatomic molecule due to collisions with an atom was explored by performing quasi-classical trajectory calculations on $\mathrm{N}_{2}-\mathrm{He}$ and $\mathrm{N}_{2}-\mathrm{Ne}$ systems governed by a sum of pairwise atom-atom Morse interactions. It is found that the conversion of the orbital angular momentum into the angular momentum of the molecule takes place when the colliding atom is very close to the classical turning point ellipsoid. The quantitative measures to define such closeness were introduced. Further, it is observed that nearly $50 \%$ of the total angular momentum transfer takes place when the colliding atom approaches the classical turning point ellipsoid and the remaining $50 \%$ transfer takes place while the atom bounces back.

PACS numbers: $34.50 . \mathrm{Ez}$

\section{Introduction}

The chemical reactions or vibrational transitions during the molecular collisions are usually associated with rotational energy transfer. Even in the processes involving the transfer of very low energy, such as molecular collisions in the interstellar space, the rotational energy transfer plays an important role. In recent years the determination of the rotationally inelastic cross-sections and rate constants by theoretical as well as experimental methods have gained new dimensions [1-7]. However, the understanding of the mechanism of the rotational energy transfer is still not well understood and remains a subject of current interest [1, 8-11].

The success of the scaling and fitting laws such as the power-gap law $[12,13]$ and the exponential-gap law [14] that attempt to predict a large number of cross-sections in terms of a few fitting parameters is well known. However, the relationship of these fitting parameters with the mechanism and the intermolecular potential parameters is not well understood.

Recently, Agrawal et al. [8] have attempted to correlate one fitting parameter of the power-gap law with the intermolecular attraction by employing the hard ellipsoid potential model. This model considers the mechanism of the conversion 
of the orbital angular momentum into the molecular angular momentum at the hard ellipsoid potential surface [11, 15-18]. One of the predictions of this model, the maximum limit of the rotational energy transfer, has been verified by these authors for a large number of situations for $\mathrm{N}_{2}-\mathrm{He}$ and $\mathrm{N}_{2}-\mathrm{Ne}$ systems. However, for understanding more about the mechanism of the rotational energy transfer an important question that requires attention is to explore the region in which most of the transfer of orbital angular momentum into molecular angular momentum takes place and to see how close is this region with the hard ellipsoid potential surface, i.e., classical turning point surface.

In this paper we intend to investigate this problem by employing the classical trajectory methods for $\mathrm{N}_{2}-\mathrm{He}$ and $\mathrm{N}_{2}-\mathrm{Ne}$ systems. The next section gives the details of the computation and results are then presented and discussed in the final section.

\section{Computational method}

\subsection{Potential energy surface}

The potential energy of the system $V$ is taken as a sum of the pairwise atom-atom interactions

$$
V=\sum_{i>j} V_{i j} \quad(i, j=1-3)
$$

where

$$
V_{i j}=D_{i j}\left\{\exp \left[-2 \alpha_{i j}\left(R_{i j}-R_{i j 0}\right)\right]-2 \exp \left[-\alpha_{i j}\left(R_{i j}-R_{i j 0}\right)\right]\right\}+D_{i j},
$$

and where $R_{12}, R_{13}$ and $R_{23}$ are the $\mathrm{N}^{(1)}-\mathrm{N}^{(2)}, \mathrm{N}^{(1)}-\mathrm{X}$, and $\mathrm{N}^{(2)}-\mathrm{X}$ distances, respectively, as shown in Fig. 1 . The potential parameters $D_{i j}, \alpha_{i j}$, and $R_{i j 0}$ are given in Table $\mathrm{I}$.

\section{TABLE I}

Potential parameters (Ref. [19]).

\begin{tabular}{l|c|c|l}
\hline \hline Atom pair & $D[\mathrm{eV}]$ & $\alpha\left[\AA^{-1}\right]$ & $R_{i j 0}[\AA]$ \\
\hline $\mathrm{N}-\mathrm{N}$ & 9.756 & 5.4844 & 1.094 \\
$\mathrm{~N}-\mathrm{Ne}$ & 0.005352 & 1.6529 & 3.63 \\
$\mathrm{~N}-\mathrm{He}$ & 0.002328 & 1.6690 & 3.595
\end{tabular}

\subsection{Trajectory calculations}

Standard classical trajectory method described in the literature [20] was used. Hamilton's equations of motion were integrated by using fourth-order Runge-Kutta method. One time step equal to 0.25 time unit ( 1 time unit $\left.=1.019 \times 10^{-14} \mathrm{~s}\right)$ was found to yield the desired accuracy in the energy conservation.

The molecule is initially considered in the rotational ground state. 1000 trajectories were run at each energy for each system.

The angular momentum of the molecule is monitored as a function of the location and orientation of the molecule and atom for each trajectory. The maximum amount of angular momentum gain, $L_{\max }$, by the molecule is evaluated for 
each trajectory. The stored information for each trajectory is used to get the location of the atom relative to the center of mass of the molecule and molecular axis whenever the angular momentum of the molecule is $f L_{\max }$ for a particular value of $f$. This information is also used to obtain the distance of the atom from the classical turning point surface. The classical turning point surface for each system at each energy is found to be very close to an ellipsoid [8]. For different values of $f$ varying from 0.1 to 0.9 this analysis is repeated.

\section{Results and discussion}

Figure 2 shows molecular axis, classical turning point surface and location of atoms corresponding to $f=0.70$ and energy $=0.05 \mathrm{eV}$. Each dot in the figure corresponds to the location of $\mathrm{Ne}$ in a trajectory in the body-frame of the $\mathrm{N}_{2}$ molecule when its angular momentum is $0.70 L_{\max }$, where $L_{\max }$ is the maximum amount of angular momentum gain by the molecule for that trajectory. Table II shows that $80 \%$ of these dots are at a distance smaller or equal to $0.432 \AA$ from the classical turning point surface. For further reference we denote this distance $R_{\max }(f=0.7, P=80 \%)$. The average distance of Ne atom from the classical turning point surface for this case, $f=0.7, E=0.05 \mathrm{eV}$, averaged over all trajectories is $0.288 \AA$.

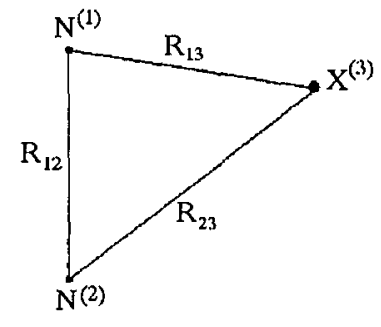

Fig. 1

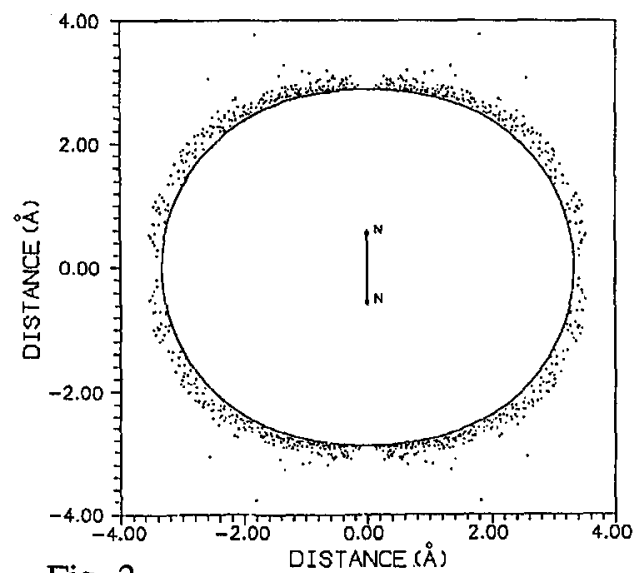

Fig. 2

Fig. 1. Coordinates for $\mathrm{N}_{2}-\mathrm{X}$ system.

Fig. 2. Classical turning point surface, the molecular axis and the location of $\mathrm{Ne}$ atoms represented by dots corresponding to $f=0.7$ and $E=0.05 \mathrm{eV}$ for $\mathrm{N}_{2}-\mathrm{Ne}$ system.

The variation of $R_{\max }$ with $f$ for this value of $P=80 \%$ is shown in Table II. For $P=90 \%$ the same is shown in Fig. 3 . The values of $R_{\max }$ listed in Table II for $E=0.05 \mathrm{eV}$ reveal that for $f=0.10, R_{\max }=0.576 \AA$. As $f$ increases, i.e. the amount of angular momentum transfer increases, $R_{\max }$ first decreases, i.e. $\mathrm{Ne}$ atoms come closer to the classical turning point surface, up to $f$ nearly equal to 0.50 , and then it starts increasing. This behavior as exhibited by the data of this Table and Fig. 3 suggests that nearly $50 \%$ angular momentum transfer takes place when the atom approaches the classical turning point surface 
TABLE II

The proximity with the classical turning point ellipsoid as a function of angular momentum transfer for $\mathrm{N}_{2}-\mathrm{Ne}$ system.

\begin{tabular}{c|c|c|c|c|c|c}
\hline \hline$f$ & \multicolumn{3}{|c|}{$E=0.05 \mathrm{eV}^{a}$} & \multicolumn{3}{c}{$E=0.10 \mathrm{eV}^{b}$} \\
\cline { 2 - 7 } & $A^{\prime}-A[\AA]$ & $B^{\prime}-B[\AA]$ & $R_{\max }^{c}[\AA]$ & $A^{\prime}-A[\AA]$ & $B^{\prime}-B[\AA]$ & $R_{\max }^{c}[\AA]$ \\
\hline 0.1 & $0.336 \pm 0.022$ & $0.359 \pm 0.007$ & 0.576 & $0.464 \pm 0.058$ & $0.446 \pm 0.019$ & 0.705 \\
0.2 & $0.244 \pm 0.022$ & $0.247 \pm 0.007$ & 0.485 & $0.368 \pm 0.070$ & $0.348 \pm 0.023$ & 0.595 \\
0.3 & $0.197 \pm 0.025$ & $0.201 \pm 0.008$ & 0.446 & $0.339 \pm 0.066$ & $0.277 \pm 0.021$ & 0.559 \\
0.4 & $0.177 \pm 0.023$ & $0.168 \pm 0.007$ & 0.418 & $0.389 \pm 0.068$ & $0.203 \pm 0.020$ & 0.536 \\
0.5 & $0.188 \pm 0.022$ & $0.143 \pm 0.006$ & 0.416 & $0.374 \pm 0.050$ & $0.191 \pm 0.014$ & 0.527 \\
0.6 & $0.206 \pm 0.021$ & $0.145 \pm 0.006$ & 0.412 & $0.535 \pm 0.108$ & $0.201 \pm 0.028$ & 0.560 \\
0.7 & $0.272 \pm 0.032$ & $0.165 \pm 0.009$ & 0.432 & $0.691 \pm 0.095$ & $0.164 \pm 0.023$ & 0.594 \\
0.8 & $0.366 \pm 0.032$ & $0.213 \pm 0.008$ & 0.469 & $0.764 \pm 0.082$ & $0.227 \pm 0.019$ & 0.669 \\
\hline
\end{tabular}

${ }^{a} A=3.3318 \AA, B=2.8814 \AA .{ }^{b} A=3.1652 \AA, B=2.7282 \AA .{ }^{c}$ For $P=80 \%$.

and the next $50 \%$ angular momentum transfer takes place when the atom bounces back from the molecule. The magnitude of this distance shows that the transfer of angular momentum from $0.1 L_{\max }$ to $0.80 L_{\max }$ takes place within a distance $0.576 \AA$ from the classical turning point surface.

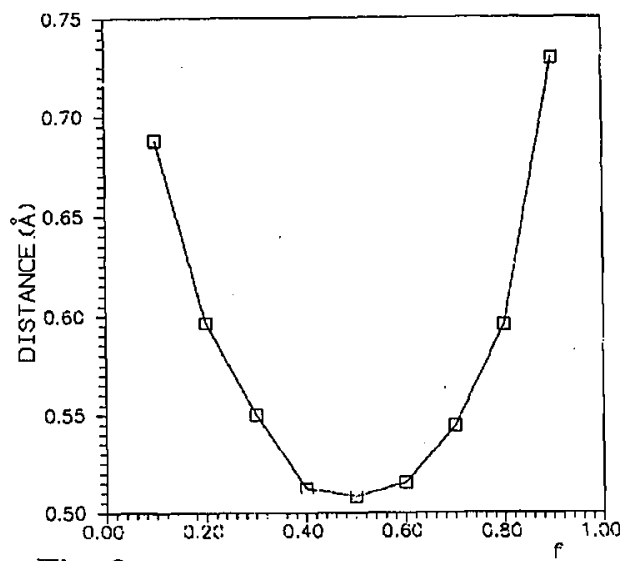

Fig. 3

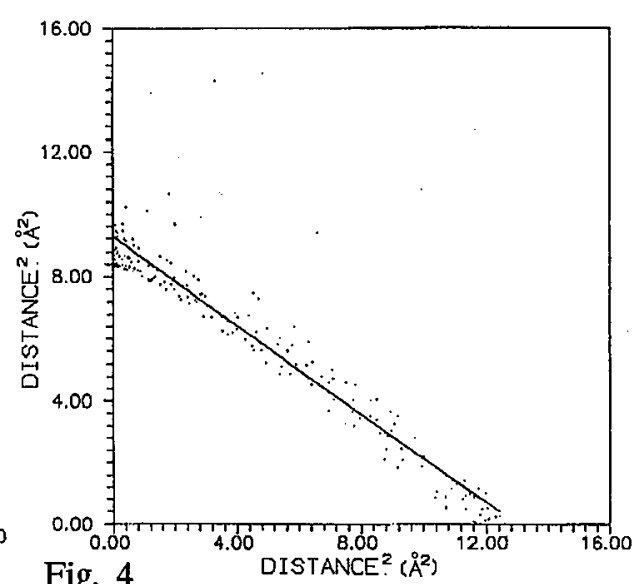

Fig. 4

Fig. 3. $R_{\max }(f, P=90 \%)$ in $\AA$ as a function of $f$ for $\mathrm{N}_{2}-\mathrm{Ne}$ system at $E=0.05 \mathrm{eV}$. Fig. 4. $X^{\prime}$ versus $Y^{\prime}$ in $\AA^{2}$ for $\mathrm{N}_{2}-\mathrm{Ne}$ corresponding to $E=0.05 \mathrm{eV}$, and $f=0.7$. The straight line shows the least squares fit.

Another quantitative measure to see the location and spread of the dots shown in Fig. 2 is to evaluate the variation in length of the semimajor and semiminor axes of the ellipse on which all these points are supposed to lie.

Let the equation of the ellipse on which a point (a dot of Fig. 2) lies be $x^{2} / A^{\prime 2}+y^{2} / B^{\prime 2}=1$ 
if we define

$$
X^{\prime}=x^{2} \quad \text { and } \quad Y^{\prime}=y^{2},
$$

then Eq. (3) becomes

$$
X^{\prime} / A^{\prime 2}+Y^{\prime} / B^{\prime 2}=1,
$$

i.e., an equation of a straight line in $X^{\prime}-Y^{\prime}$ space. The $X^{\prime}-Y^{\prime}$ plot of all the points shown by dots in Fig. 2 is shown in Fig. 4. The least squares fit of these points shown in Fig. 4 corresponds to $A^{\prime}=3.6043$ and $B^{\prime}=3.0467$ with the fluctuation $\Delta A^{\prime}=0.032$ and $\Delta B^{\prime}=0.009$. This shows that the locus of all points corresponding to the transfer of angular momentum equal to $f L_{\max }$ is an ellipse for the collision in a plane.

For different values of $f$ these results are shown in Table II. Here again we see that the departure of $A^{\prime}$ and $B^{\prime}$ from the respective lengths of the semimajor and semiminor axes, $A$ and $B$, corresponding to classical turning point surface is small (smaller than $0.37 \AA$ ). It shows that the angular momentum transfer takes place in the region very close to the classical turning point surface.

These conclusions are also confirmed by the data listed in Table II for $E=$ $0.10 \mathrm{eV}$ for $\mathrm{N}_{2}-\mathrm{Ne}$ and those shown in Table III for $\mathrm{N}_{2}-\mathrm{He}$ at $E=0.20 \mathrm{eV}$.

\section{TABLE III}

The proximity with the classical turning point ellipsoid as a function of angular momentum transfer for $\mathrm{N}_{2}-\mathrm{He}$ system.

\begin{tabular}{c|c|c|c}
\hline \hline$f$ & \multicolumn{3}{|c}{$E=0.2 \mathrm{eV}^{a}$} \\
\cline { 2 - 4 } & $A^{\prime}-A[\AA]$ & $B^{\prime}-B[\AA]$ & $R_{\max }^{b}[\AA]$ \\
\hline 0.1 & $0.456 \pm 0.075$ & $0.244 \pm 0.020$ & 0.616 \\
0.2 & $0.389 \pm 0.073$ & $0.156 \pm 0.019$ & 0.566 \\
0.3 & $0.368 \pm 0.074$ & $0.104 \pm 0.019$ & 0.528 \\
0.4 & $0.615 \pm 0.128$ & $0.006 \pm 0.027$ & 0.513 \\
0.5 & $0.375 \pm 0.072$ & $0.075 \pm 0.018$ & 0.506 \\
0.6 & $0.387 \pm 0.070$ & $0.099 \pm 0.018$ & 0.507 \\
0.7 & $0.636 \pm 0.152$ & $0.093 \pm 0.034$ & 0.546 \\
0.9 & $0.597 \pm 0.059$ & $0.395 \pm 0.016$ & 0.762 \\
\hline${ }^{a} A=2.7476 \AA, B=2.3196 \AA .{ }^{b}$ For $P=80 \%$.
\end{tabular}

All these results further widen the applicability of the hard ellipsoid potential model. It also leads to the requirement of focusing our attention for the interaction potential near the classical turning point surface for the purpose of studying the rotational energy transfer.

Further studies are in progress to determine the dependence of $R_{\max }$ on $L_{\max }$, potential parameters, collision energy, rotational constant of the molecule and other dynamical parameters. Such a study is expected to lead to the better 
understanding of the mechanism of the rotational energy transfer and the explanation of the rotational inelasticity in terms of the knowledge of the classical turning point ellipsoid and $R_{\max }$. This may eventually also lead to the understanding of the success of the scaling and fitting laws [12-14, 21,22] that describe the rotationally inelastic state-to-state cross-sections in terms of a few fitting parameters.

\section{Acknowledgment}

Authors are thankful to Dr. P.M. Agrawal for his kind guidance to pursue this research.

\section{References}

[1] T.W.J. Whitely, A.J. McCaffery, J. Phys. B 29, 6133 (1996).

[2] F.A. Gianturco, S. Kumar, J. Phys. B 30, 3031 (1997).

[3] F.A. Gianturco, S. Kumar, S.K. Pathak, M. Raimondi, M. Sironi, Chem. Phys. 215, 239 (1997).

[4] P.M. Agrawal, N.K. Dabkara, S. Tilwankar, Chem. Phys. Lett. 266, 481 (1997).

[5] P.L. James, I.R. Sims, I.W.M. Smith, Chem. Phys. Lett. 272, 412 (1997).

[6] C.C. Wang, T.L. Chin, K.C. Lin, J. Chem. Phys. 107, 10348 (1997).

[7] G. Millot, C. Roche, J. Raman. Spectrosc. 29, 313 (1998).

[8] P.M. Agrawal, S. Tilwankar, N.K. Dabkara, J. Chem. Phys. 108, 4854 (1998).

[9] M.A. Osborne, A.J. McCaffery, J. Chem. Phys. 101, 5604 (1994).

[10] A.J. McCaffery, Z.T. Alwahabi, M.A. Osborne, C.J. Williams, J. Chem. Phys. 98, 4586 (1993).

[11] F.A. Gianturco, J.P. Toennies, M. Bernardi, J. Chem. Soc. Faraday Trans. 87, 31 (1991).

[12] T.A. Burner, N. Smith, A.W. Karp, D.E. Pritchard, J. Chem. Phys. 74, 3324 (1981).

[13] I. NoorBatcha, N. Sathyamurthy, Chem. Phys. Lett. 79, 264 (1981).

[14] J.C. Polanyi, K.B. Woodall, J. Chem. Phys. 56, 1563 (1972).

[15] A.J. McCaffery, Z.T. Alwahami, Phys. Rev. A 43, 611 (1991).

[16] S. Bosanac, Phys. Rev. A 22, 2617 (1980).

[17] D. Beck, U. Ross, W. Schepper, Z. Phys. A 293, 107 (1979).

[18] J.A. Serri, R.M. Billota, D.E. Pritchard, J. Chem. Phys. 77, 2940 (1982).

[19] (a) American Institute of Physics Handbook, Ed. D.E. Gray, McGraw Hill, New York 1957, p. 4.129, the geometric mean rule for $\varepsilon$ and arithmetic mean rule for $\sigma$ have been used; the parameters of $L-J$ potential have been converted into parameters of the Morse potential by taking the same location of the minima and the same second derivative at the minima; (b) R.A. Svella, NASA Tech. Rept. TR R-132 (1962).

[20] L.M. Raff, D.L. Thompson, in: Theory of Chemical Reaction Dynamics, Vol. III, Ed. M. Baer, CRC, Boca Raton (FL) 1985, p. 1.

[21] B.J. Whitaker, Ph. Brechignac, Chem. Phys. Lett. 95, 407 (1983).

[22] R. Ramaswamy, A.E. DePristo, H. Rabitz, Chem. Phys. Lett. 61, 495 (1979). 